\title{
Parameter Control of Stochastic Neural Network System with Markov Switching
}

\author{
Bo Hong (iD) and Hui Wang (10) \\ School of Computer Science and Engineering, Xi'an Technological University, Xi'an 710021, China \\ Correspondence should be addressed to Hui Wang; wanghuihy@xatu.edu.cn
}

Received 22 October 2021; Revised 22 November 2021; Accepted 3 January 2022; Published 22 January 2022

Academic Editor: Narasimhan Venkateswaran

Copyright (c) 2022 Bo Hong and Hui Wang. This is an open access article distributed under the Creative Commons Attribution License, which permits unrestricted use, distribution, and reproduction in any medium, provided the original work is properly cited.

\begin{abstract}
The stochastic control of Markov switching systems with time-delay feedback neural networks under the interference of external environment is studied in this paper. By designing a memoryless state feedback controller, a set of sufficient conditions for the stochastic stability of the switching system and the disturbance attenuation are obtained by using the stochastic assessment method and the linear matrix inequality, and the key $H_{\infty}$ control of this problem is realized.
\end{abstract}

\section{Introduction}

Artificial neural network is a developed and mature high technology in recent decades. The research of the neural network has achieved great success. The network has been widely used in the fields of image and television signal processing, robot and biological vision, advanced brain function, and so on. In fact, as the most basic structural and functional unit of the nervous system, the neurons have the function of connecting and integrating input information and transmitting information. The signal transmission in the neurons is not simple and mechanical reception and transmission, but it needs the signal to reach a threshold before generating the output signal, that is, the neurons receive the signal, and then, the signal is processed and transmitted to the next neuron. In practical application, the information transmission between the interacting neurons takes a certain time, so the phenomenon with the time delay is inevitable [1-4]. The existence of the time delay tends to cause the network oscillation, bifurcation, or chaos and even cause the network instability. In the real nervous system, the transmission of information between the neurons is a complex process with the noise, because the transmission medium and the signal itself will be affected by the random fluctuation of the environment. Therefore, it is very important to study the influence of random interference on the stability of the neural networks [5-8].

In recent years, there have been a lot of research results [9-12]. In reference [8], Cao used Young's inequality technique to give the sufficient conditions for the exponential stability of the equilibrium point of the delayed neural network system and discussed the stability of the periodic solution. Chen analyzed the exponential stability of the time-delay neural network system without requiring the activation function to be bounded in reference [9]. In the real nervous system, the transmission of information between the synapses is a complex noisy process, because both the transmission medium and the signal itself are affected by the random fluctuations of the environment [13-16]. There are also many achievements in the research of random neural network, such as Wan and Sun [17-19]; Sun and Cao [20] studied the mean square and $p$-order moment stability of delay neural networks by using the method of variation coefficient. Besides, Wang et al. derived the asymptotic stability conditions of Cohen-Grossberg neural networks by using the Lyapunov method [21].

However, there are many modes in the operation of neural network, which can be switched according to the situation. Some studies have shown that this mode transformation of the neural network can be described by homogeneous 
Markov chain [22], so the neural network systems with Markov switching have become one of the focuses of the neural network research. Markov switching system can be used to describe the sudden transition of the system state, such as the random failure and repair of the system components, the change of the subsystem connection or interaction mode of the complex system, and the change of environmental factors [23-28]. In this paper, we will study the problems of stochastic robust stabilization and robust $H_{\infty}$ control for switched time-delay feedback neural network systems that are subject to external environmental disturbance inputs. Considering the influence of disturbance input, when the appropriate linear matrix inequality conditions are satisfied, the system is robustly stable under feedback control and achieves the given $H_{\infty}$ index. For delayed neural networks with Markov jump parameters, a memoryless state feedback controller is designed to achieve similar stabilization and $H_{\infty}$ control objectives.

\section{Problem Description}

Let a Markov process defined on state space $S=\{1,2, \cdots, N\}$ be $\{\varphi(t), t \geq 0\}$, and the state transition-rate matrix is

$$
P\left(\varphi_{i j}(t+\Delta l)=j \mid \varphi(t)=i\right)=\left\{\begin{array}{l}
r_{i j} \Delta l+o(\Delta l), \quad i \neq j, \\
1+r_{i i} \Delta l+o(\Delta l), \quad i=j,
\end{array}\right.
$$

where $\Delta l$ is the uncertainty matrix of corresponding parameter $t$ and $\varphi_{i j} \geq 0(i \neq j)$ is the transition rate from state $i$ to $j$.

$$
\varphi_{i i}=-\sum \varphi_{i j},(i \neq j), \quad \text { also } \quad \Delta l>0, \lim _{\Delta l \longrightarrow 0} \frac{o(\Delta l)}{\Delta l}=0 \text {. }
$$

Now, the following uncertain systems of delayed stochastic neural network with Markov jump parameters are given as follows:

$$
\left\{\begin{array}{l}
\mathrm{d} x(t)=\left[A(\varphi(t)) f(x(t))+A_{\partial}(\varphi(t)) f(x(t-\tau))+B_{\mu}(\varphi(t)) \mu(t, \varphi(t))+B_{v}(\varphi(t)) v(t)-C(\varphi(t)) x(t)\right] d t+\left[E(\varphi(t)) x(t)+E_{\partial}(\varphi(t)) x(t-\tau)\right] \mathrm{d} \omega(t) \\
y(t)=D(\varphi(t)) x(t)+D_{\mu}(\varphi(t)) \mu(t, \varphi(t)) \\
x(t)=\vartheta(t), \varphi(t)=\varphi(0), \quad \forall t \in[-\tau, 0], \vartheta(t) \in C\left([-\tau, 0] ; \mathbb{R}^{n}\right)
\end{array}\right.
$$

where $f:\left(\mathbb{R}^{+} \times \mathbb{R}^{n} \longrightarrow \mathbb{R}^{n}\right)$ is the Borel measurable function, which is similar to $\mathbb{R}^{n}$ expressed as $n$-dimensional Euclidean space. $x(t)=\left(x_{1}(t), \cdots, x_{n}(t)\right)^{T}$ is $N$ neuron state vector, $\mu(t, \varphi(t)) \in \mathbb{R}^{\varphi}$ is control inputs, and $v(t) \in \mathbb{R}^{p}$ is interference inputs, satisfying $v(t) \in L_{2}[0, \infty) . y(t) \in \mathbb{R}^{q}$ is the control outputs, $\tau$ is a constant with time delay and $\tau>0, \omega(t)$ is a one-dimensional Brownian motion satisfying $\mathbb{E}[d \omega(t)]=0$ and $\mathbb{E}[d \omega(t)]^{2}=d t$, which is similar to $\mathbb{E}$ (.), and it represents the mathematical expectation. Besides, $\vartheta(t)$ is the initial condition of the function space defined on $[-\tau, 0]$ and valued at $\mathbb{R}^{n} . A(\varphi(t)), A_{\partial}(\varphi(t))$, $B_{\mu}(\varphi(t)), B_{v}(\varphi(t)), C(\varphi(t)), E(\varphi(t)), E_{\partial}(\varphi(t)), D(\varphi(t))$, and $D_{\mu}(\varphi(t))$ are all constant matrices of appropriate dimensions.

In addition, for the state $\varphi(t)=i$ of Markov chain, it is abbreviated as $\Phi_{i}={ }^{\Delta l} \Phi(\varphi(t)=i)$, and $\Phi$ represents the matrix in the system. Similar to reference [29], the following assumptions for the activation function defined to run on the neurons of the artificial neural network can be given.

The activation function is bounded and must satisfy the Lipschitz condition. That is, there is a constant matrix $P \in$ $R^{n \times n}$, which satisfies

$$
|f(\mu)-f(v)| \leq|P(\mu-v)|, \quad \forall \mu, v \in \mathbb{R}^{n}
$$

where $x(t ; \psi)$ is the system state track starting from the initial value $x(s)=\psi(s), s \in[-\tau, 0]$, and $\psi($.$) belongs to$ $F_{0}$ measurable twice integrable $C\left([-\tau, 0] ; \mathbb{R}^{n}\right)$ random process space. That is, $L_{F_{0}}^{2}\left([-\tau, 0] ; \mathbb{R}^{n}\right)$. Obviously, for the initial value $\psi=0, x(t) \equiv 0$ is the trivial solution of system (3).

Lemma 1 [30]. For any given matrix,

$$
A=\left(\begin{array}{ll}
A_{11} & A_{12} \\
A_{12}^{T} & A_{22}
\end{array}\right)<0
$$

where $A_{11}=A_{11}^{T}$ and $A_{22}=A_{22}^{T}$; it is equivalent to

$$
\begin{array}{ll}
A_{11}<0, & A_{22}-A_{12}^{T} A_{11}^{-1} A_{12}<0, \\
A_{22}<0, & A_{11}-A_{12}^{T} A_{22}^{-1} A_{12}<0 .
\end{array}
$$

\section{Main Results}

Theorem 1. Considering system (3), for $v(t)=0$, if there is a constant $\varepsilon>0$, the positive definite matrices $X_{i}>0$ and $M>0$ constant matrix $C_{i}$ and matrix $Y_{i}$ such that the linear matrix inequality (LMI) $g<0$ holds for all $i \in S$, 
then the system (3) is stochastic robust stabilization by the feedback controller $\mu(t, i)$.

$$
\begin{gathered}
g=\left(\begin{array}{cccccccc}
\mathfrak{R}_{i} & 0 & \varepsilon A_{i} & \varepsilon A_{\partial i} & X_{i} E_{i}^{T} & X_{i} P^{T} & X_{i} & \pi_{i} \\
0 & -M & 0 & 0 & M E_{\partial i}^{T} & 0 & 0 & 0 \\
\varepsilon A_{i}^{T} & 0 & -\varepsilon I & 0 & 0 & 0 & 0 & 0 \\
\varepsilon A_{\partial i}^{T} & 0 & 0 & -\varepsilon I & 0 & 0 & 0 & 0 \\
E_{i} X_{i} & E_{\partial i} M & 0 & 0 & -X_{i} & 0 & 0 & 0 \\
P X_{i} & 0 & 0 & 0 & 0 & -\frac{1}{2} \varepsilon I & 0 & 0 \\
X_{i} & 0 & 0 & 0 & 0 & 0 & -M & 0 \\
\pi_{i}^{T} & 0 & 0 & 0 & 0 & 0 & 0 & J_{i}
\end{array}\right), \\
\mathfrak{R}_{i}=B_{\mu i} Y_{i}+Y_{i}^{T} B_{\mu i}^{T}+\left(\varphi_{i i}-C_{i}\right) X_{i}-X_{i} C_{i}, \\
\pi_{i}=\left[X_{i}, \cdots, X_{i}\right]_{n \times(N-1) n}, \\
J_{i}=\operatorname{diag}\left\{\pi_{i 1}^{-1} X_{1}, \cdots, \pi_{i i-1}^{-1} X_{i-1}, \pi_{i i+1}^{-1} X_{i+1}, \cdots, \pi_{i N}^{-1} X_{N}\right\} \\
\mu(t, i)=k_{i} x(t), k_{i}=Y_{i} X_{i}^{-1} .
\end{gathered}
$$

Proof. By using the feedback controller $\mu(t, i)$ to the system (3) with $v(t)=0$, the following closed-loop system can be obtained.

$$
\begin{aligned}
d x(t)= & {\left[A_{i} f(x(t))+A_{\partial i} f(x(t-\tau))+\left(B_{\mu i} k_{i}-C_{i}\right) x(t)\right] d t } \\
& +\left[E_{i} x(t)+E_{\partial i} x(t-\tau) d \omega(t) .\right.
\end{aligned}
$$

Now, the Lyapunov functional is selected as follows.

$$
\begin{aligned}
V(t)= & x^{\mathrm{T}}(t) X_{i}^{-1} x(t)+\int_{t-\tau}^{t} x^{\mathrm{T}}(s) M^{-1} x(s) d s \\
& +\int_{t-\tau}^{t} f^{\mathrm{T}}(x(s))\left(\varepsilon^{-1} I\right) f(x(s)) d s .
\end{aligned}
$$

By using the Itô formula, it can be concluded

$$
d V(t)=\mathfrak{Q} V(t) d t+2 x^{\mathrm{T}}(t) X_{i}^{-1}\left[E_{i} x(t)+E_{\partial i} x(t-\tau)\right] d \omega(t),
$$

where

$$
\begin{aligned}
\mathfrak{Q} V(t)= & 2 x^{\mathrm{T}}(t) X_{i}^{-1}\left[A_{i} f(x(t))+A_{\partial i} f(x(t-\tau))+\left(B_{\mu i} k_{i}-C_{i}\right) x(t)\right] \\
& +\sum_{j=1}^{N} \varphi_{i j} x^{\mathrm{T}}(t) X_{j}^{-1} x(t)+\left[E_{i} x(t)+E_{\partial i} x(t-\tau)\right] X_{i}^{-1} \\
& \cdot\left[E_{i} x(t)+E_{\partial i} x(t-\tau)\right]+x^{\mathrm{T}}(t) M^{-1} x(t)-x^{\mathrm{T}}(t-\tau) \\
& \cdot\left(\varepsilon^{-1} I\right) x(t-\tau)+f^{\mathrm{T}}(x(t))\left(\varepsilon^{-1} I\right) f(x(t))-f^{\mathrm{T}}(x(t-\tau)) \\
& \cdot\left(\varepsilon^{-1} I\right) f(x(t-\tau)) .
\end{aligned}
$$

According to the assumption (4), it can be concluded

$$
-2 \varepsilon^{-1}\left[f^{\mathrm{T}}\left(x(t) f(x(t))-x^{\mathrm{T}}(t) P^{\mathrm{T}}(x(t)) P(x(t))\right] \geq 0 .\right.
$$

Comprehensive (11) and (12), it can be concluded

$$
\mathfrak{Q} V(t) \leq \phi^{\mathrm{T}}(t) \mathcal{Y}_{i} \phi(t)
$$

where

$$
\begin{gathered}
\Upsilon_{i}=\left(\begin{array}{cccc}
-\Upsilon_{1 i} & 0 & X_{i}^{-1} A_{i} & X_{i}^{-1} A_{\partial i} \\
0 & -M^{-1} & 0 & 0 \\
A_{i}^{\mathrm{T}} X_{i}^{-1} & 0 & -\varepsilon^{-1} I & 0 \\
A_{\partial i}^{\mathrm{T}} X_{i}^{-1} & 0 & 0 & -\varepsilon^{-1} I
\end{array}\right)+\left[\begin{array}{c}
E_{i}^{\mathrm{T}} \\
E_{\partial i}^{\mathrm{T}} \\
0 \\
0
\end{array}\right] X_{i}^{-1}\left[E_{i} E_{\partial i} 00\right], \\
\Upsilon_{1 i}=X_{i}^{-1}\left(C_{i}-B_{\mu i} k_{i}\right)-\left(C_{i}-k_{i}^{\mathrm{T}} B_{\mu i}^{\mathrm{T}}\right) X_{i}^{-1}-M^{-1}-\sum_{j=1}^{N} \varphi_{i j} X_{j}^{-1}, \\
\phi^{\mathrm{T}}(t)=x^{\mathrm{T}}(t) x^{\mathrm{T}}(t-\tau)(x(t)) f^{\mathrm{T}}(x(t-\tau)) .
\end{gathered}
$$

Multiplying the both sides of item for inequality $g<0$ by $\operatorname{diag}\left\{X_{i}^{-1}, M^{-1}, \varepsilon^{-1} I, \varepsilon^{-1} I, I, I, I\right\}$, it can be concluded

$$
\left(\begin{array}{cccccccc}
\Re_{i}{ }^{\prime} & 0 & X_{i}^{-1} A_{i} & X_{i}^{-1} A_{\partial i} & E_{i}^{\mathrm{T}} & P^{\mathrm{T}} & I & \pi_{i} \\
0 & -M & 0 & 0 & E_{\partial i}^{\mathrm{T}} & 0 & 0 & 0 \\
A_{i}^{\mathrm{T}} X_{i}^{-1} & 0 & -M^{-1} I & 0 & 0 & 0 & 0 & 0 \\
A_{\partial i}^{\mathrm{T}} X_{i}^{-1} & 0 & 0 & -M^{-1} I & 0 & 0 & 0 & 0 \\
E_{i} & E_{\partial i} & 0 & 0 & -X_{i} & 0 & 0 & 0 \\
P & 0 & 0 & 0 & 0 & -\frac{1}{2} \varepsilon I & 0 & 0 \\
I & 0 & 0 & 0 & 0 & 0 & -M & 0 \\
\pi_{i}^{\mathrm{T}} & 0 & 0 & 0 & 0 & 0 & 0 & J_{i}
\end{array}\right)<0,
$$

where

$$
\begin{gathered}
\mathfrak{R}_{i}{ }^{\prime}=\left(\varphi_{i i}+k_{i}^{\mathrm{T}} B_{\mu i}^{\mathrm{T}}-C_{i}\right) X_{i}^{-1}+X_{i}^{-1}\left(B_{\mu i} k_{i}-C_{i}\right), \\
\pi_{i}=[I, \cdots I]_{n \times(N-1) n} .
\end{gathered}
$$

From Lemma 1, it can be seen that formula (15) holds $r_{i}<0$. According to equation (13), if $\phi^{\mathrm{T}}(t) \neq 0$ holds,

$$
\mathfrak{Q} V(t)<0 \text {. }
$$

Then, the trivial solution of closed-loop system (8) can be derived from Lyapunov stability theory, which is asymptotically stable in the mean square sense. That is, the system (3) is robust stabilization under $\mu(t, i)=k_{i} x(t)$ (where $k_{i}=Y_{i}$ $X_{i}^{-1}$ ) state feedback control.

Hence, the proof ends.

Next, a set of sufficient conditions for $H_{\infty}$ control of uncertain systems (3) with time-delay stochastic neural networks with Markov jump parameters are given in the form of theorems. 
Theorem 2. Considering the case of nonzero interference input $v(t) \in L_{2}[0, \infty)$, for the system (3), if there are constant $\varepsilon>0$, given constant $\beta$, positive definite matrices $X_{i}>0$ and $M>0$, constant matrix $C_{i}$ and matrix $Y_{i}$, such that the linear matrix inequality (LMI) $G<0$ holds for all $i \in S$, then the system (3) is stochastic robust stabilization by the feedback controller $\mu(t, i)$.

$$
G=\left(\begin{array}{cccccccccc}
\Re_{i} & 0 & \varepsilon A_{i} & \varepsilon A_{\partial i} & X_{i} E_{i}^{T} & X_{i} P^{T} & X_{i} & \pi_{i} & B_{v i} & \Omega_{i} \\
0 & -M & 0 & 0 & M E_{\partial i}^{T} & 0 & 0 & 0 & 0 & 0 \\
\varepsilon A_{i}^{T} & 0 & -\varepsilon I & 0 & 0 & 0 & 0 & 0 & 0 & 0 \\
\varepsilon A_{\partial i}^{T} & 0 & 0 & -\varepsilon I & 0 & 0 & 0 & 0 & 0 & 0 \\
E_{i} X_{i} & E_{\partial i} M & 0 & 0 & -X_{i} & 0 & 0 & 0 & 0 & 0 \\
P X_{i} & 0 & 0 & 0 & 0 & -\frac{1}{2} \varepsilon I & 0 & 0 & 0 & 0 \\
X_{i} & 0 & 0 & 0 & 0 & 0 & -M & 0 & 0 & 0 \\
\pi_{i}^{T} & 0 & 0 & 0 & 0 & 0 & 0 & J_{i} & 0 & 0 \\
B_{v i}^{T} & 0 & 0 & 0 & 0 & 0 & 0 & 0 & -\beta^{2} I & 0 \\
\Omega_{i}^{T} & 0 & 0 & 0 & 0 & 0 & 0 & 0 & 0 & -I
\end{array}\right),
$$

$$
\begin{gathered}
\mathfrak{R}_{i}=B_{\mu i} Y_{i}+Y_{i}^{T} B_{\mu i}^{T}+\left(\varphi_{i i}-C_{i}\right) X_{i}-X_{i} C_{i}, \\
\pi_{i}=\left[X_{1}, \cdots, X_{N}\right]_{n \times(N-1) n}, \\
J_{i}=\operatorname{diag}\left\{\pi_{i 1}^{-1} X_{1}, \cdots, \pi_{i i-1}^{-1} X_{i-1}, \pi_{i i+1}^{-1} X_{i+1}, \cdots, \pi_{i N}^{-1} X_{N}\right\} \\
\Omega_{i}=C_{i} D_{i}^{T}+Y_{i}^{T} D_{\mu i}^{T}, \\
\mu(t, i)=k_{i} x(t), \quad k_{i}=Y_{i} X_{i}^{-1} .
\end{gathered}
$$

Proof. By using the feedback controller $\mu(t, i)$ to the system (3), the following closed-loop system can be obtained

$$
\left\{\begin{array}{l}
d x(t)=\left[A_{i} f(x(t))+A_{\partial i} f(x(t-\tau))+\left(B_{\mu i} k_{i}-C_{i}\right) x(t)\right] d t+\left[E_{i} x(t)+E_{\partial i} x(t-\tau)\right] d \omega(t), \\
y(t)=\left(D_{i}+D_{\mu i} k_{i}\right) x(t) .
\end{array}\right.
$$

By using Lemma 1 , the inequality $g<0$ that can be deduced by the inequality $G<0$ is tenable, so when $v$ $(t)=0$, the closed-loop system (8) is the stochastic robust stabilization.

The following proves that when the nonzero interference input $v(t) \in L_{2}[0, \infty)$, it is assumed that the system (3) satisfies the following conditions:

$$
\|y(t)\|_{\mathbb{E}_{2}}<\beta\|v(t)\|_{2} .
$$

In order to prove that (20) is true, now it can be supposed that $x(t)=0, \forall t \in[-\tau, 0]$ for the system (3) and also select the following Lyapunov functional (9), which is obtained from the Itô formula.

$$
\mathbb{E}\{V(t)\}=\mathbb{E}\left\{\int_{0}^{t} \mathfrak{Q} V(s) d s\right\},
$$

where

$$
\mathfrak{Q} V(t) \leq \phi^{\mathrm{T}}(t) \Upsilon_{i} \phi(t)+2 x^{\mathrm{T}}(t) X_{i}^{-1} B_{v i} v(t) .
$$

$\phi^{\mathrm{T}}(t)$ and $\Upsilon_{i}$ have the same meaning as (13).
Let $\mathbb{Q}(t)=\mathbb{E}\left\{\int_{0}^{t}\left[y^{\mathrm{T}}(s) y(s)-\beta^{2} v^{\mathrm{T}}(s) v(s)\right] d s\right\}$ and $t>0$, and it is easy to see from (21). The following inequality holds for $t>0$ :

$$
\begin{aligned}
\mathbb{Q}(t) & \leq \mathbb{E}\left\{\int_{0}^{t}\left[y^{\mathrm{T}}(s) y(s)-\beta^{2} v^{\mathrm{T}}(s) v(s)+\mathfrak{Q} V(x(s), s, \varphi(s))\right] d s\right\} \\
& \leq \mathbb{E}\left\{\int_{0}^{t} \rho^{\mathrm{T}}(s) \aleph_{i} \rho(s) d s\right\},
\end{aligned}
$$

where

$$
\begin{gathered}
\rho^{\mathrm{T}}(t)=x^{\mathrm{T}}(t) x^{\mathrm{T}}(t-\tau) f^{\mathrm{T}}(x(t)) f^{\mathrm{T}}(x(t-\tau)) v^{\mathrm{T}}(t), \\
\aleph_{i}=\left(\begin{array}{ccccc}
-\aleph_{1 i} & 0 & X_{i}^{-1} A_{i} & X_{i}^{-1} A_{\partial i} & X_{i}^{-1} B_{v i} \\
0 & -M^{-1} & 0 & 0 & 0 \\
A_{i}^{\mathrm{T}} X_{i}^{-1} & 0 & -\varepsilon^{-1} I & 0 & 0 \\
A_{\partial i}^{\mathrm{T}} X_{i}^{-1} & 0 & 0 & -\varepsilon^{-1} I & 0 \\
B_{v i}^{\mathrm{T}} X_{i}^{-1} & 0 & 0 & 0 & -\beta^{2} I
\end{array}\right)
\end{gathered}
$$




$$
\begin{gathered}
+\left[\begin{array}{c}
E_{i}^{\mathrm{T}} \\
E_{\partial i}^{\mathrm{T}} \\
0 \\
0 \\
0
\end{array}\right] X_{i}^{-1}\left[\begin{array}{lllll}
E_{i} & E_{\partial i} & 0 & 0 & 0
\end{array}\right], \\
\aleph_{1 i}=X_{i}^{-1}\left(C_{i}-B_{\mu i} k_{i}\right)-\left(C_{i}-k_{i}^{\mathrm{T}} B_{\mu i}^{\mathrm{T}}\right) X_{i}^{-1}-M^{-1}-\sum_{j=1}^{N} \varphi_{i j} X_{j}^{-1} \\
-\left(D_{i}+D_{\mu i} k_{i}\right)^{\mathrm{T}}\left(D_{i}+D_{\mu i} k_{i}\right) .
\end{gathered}
$$

Referring to the proof of Theorem 1, it is multiplied to the both sides of item for inequality $G<0$ by $\operatorname{diag}\left\{X_{i}^{-1}\right.$, $\left.M^{-1}, \varepsilon^{-1} I, \varepsilon^{-1} I, I, I, I, I, I\right\}$; it can be obtained

$\left(\begin{array}{cccccccccc}\Re_{i}^{\prime} & 0 & X_{i}^{-1} A_{i} & X_{i}^{-1} A_{\partial i} & E_{i}^{\mathrm{T}} & P^{\mathrm{T}} & I & X_{i}^{-1} \pi_{i} & X_{i}^{-1} B_{v i} & X_{i}^{-1} \Omega_{i} \\ 0 & -M & 0 & 0 & E_{\partial i}^{\mathrm{T}} & 0 & 0 & 0 & 0 & 0 \\ A_{i}^{\mathrm{T}} X_{i}^{-1} & 0 & -\varepsilon^{-1} I & 0 & 0 & 0 & 0 & 0 & 0 & 0 \\ A_{\partial i}^{\mathrm{T}} X_{i}^{-1} & 0 & 0 & -\varepsilon^{-1} I & 0 & 0 & 0 & 0 & 0 & 0 \\ E_{i} & E_{\partial i} & 0 & 0 & -X_{i} & 0 & 0 & 0 & 0 & 0 \\ P & 0 & 0 & 0 & 0 & -\frac{1}{2} \varepsilon I & 0 & 0 & 0 & 0 \\ I & 0 & 0 & 0 & 0 & 0 & -M & 0 & 0 & 0 \\ \pi_{i}^{\mathrm{T}} X_{i}^{-1} & 0 & 0 & 0 & 0 & 0 & 0 & J_{i} & 0 & 0 \\ B_{v i}^{\mathrm{T}} X_{i}^{-1} & 0 & 0 & 0 & 0 & 0 & 0 & 0 & -\beta^{2} I & 0 \\ \Omega_{i}^{\mathrm{T}} X_{i}^{-1} & 0 & 0 & 0 & 0 & 0 & 0 & 0 & 0 & -I\end{array}\right)<0$.
It is worth noting that the definition of $\Re_{i}$ is the same as the definition of the inequality (15) in Theorem 1. By using Lemma 1, it can be obtained

$$
\aleph_{1}<0, \quad \forall i \in S
$$

Then, the following closed-loop system is obtained for $\rho^{\mathrm{T}}(t) \neq 0$ from (23).

$$
\mathbb{Q}(t) \leq \mathbb{E}\left\{\int_{0}^{t} \rho^{\mathrm{T}}(s) \aleph_{i} \rho(s) d s\right\}<0
$$

Then, the following formula can be inferred

$$
\mathbb{E}\left\{\int_{0}^{t} y^{\mathrm{T}}(s) y(s) d s\right\}<\mathbb{E}\left\{\int_{0}^{t} \beta^{2} v^{\mathrm{T}}(s) v(s) d s\right\} .
$$

It is obvious that the equivalent is true.

$$
\|y(t)\|_{\mathbb{E}_{2}}<\beta\|v(t)\|_{2} .
$$

Therefore, the proof ends.

If the noise environment is not considered, that is, random disturbances are removed from the system (3), the following Markov switched neural network system without noise interference can be obtained.

$$
\left\{\begin{array}{l}
d^{\prime} x(t)=A(\varphi(t)) f(x(t))+A_{\partial}(\varphi(t)) f(x(t-\tau))+B_{\mu}(\varphi(t)) \mu(t, \varphi(t))+B_{v}(\varphi(t)) v(t)-C(\varphi(t)) x(t), \\
y(t)=D(\varphi(t)) x(t)+D_{\mu}(\varphi(t)) \mu(t, \varphi(t)) \\
x(t)=\vartheta(t), \quad \varphi(t)=\varphi(0), \quad \forall t \in[-\tau, 0] .
\end{array}\right.
$$

For this system, it can be directly derived an inference from Theorems 1 and 2.

Based on the above proof, the basic process of the timedelay neural network system with Markov switching is to design a memoryless state feedback control so that the system can achieve stabilization and stochastic stability about disturbance attenuation when the system meets the appropriate $L M I$ conditions, so as to realize $H_{\infty}$ control of the problem.

Inference: for stochastic uncertain systems (30), if there are constant $\varepsilon>0$, given constant $\beta$, positive definite matrices $X_{i}>0$ and $M>0$, constant matrix $C_{i}$ and matrix $Y_{i}$, such that the linear matrix inequality (LMI) $g^{\prime \prime}<0$ holds for all $i \in S$, then the system (30) is stochastic robust stabilization by the feedback controller $\mu(t, i)$.

$$
g^{\prime \prime}=\left(\begin{array}{cccccccccc}
\Re_{i} & 0 & \varepsilon A_{i} & \varepsilon A_{\partial i} & X_{i} P^{\mathrm{T}} & X_{i} & \pi_{i} & B_{v i} & \Omega_{i} & 0 \\
0 & -M & 0 & 0 & 0 & 0 & 0 & 0 & 0 & 0 \\
\varepsilon A_{i}^{\mathrm{T}} & 0 & -\varepsilon I & 0 & 0 & 0 & 0 & 0 & 0 & 0 \\
\varepsilon A_{\partial i}^{\mathrm{T}} & 0 & 0 & -\varepsilon I & 0 & 0 & 0 & 0 & 0 & 0 \\
P X_{i} & 0 & 0 & 0 & -\frac{1}{2} \varepsilon I & 0 & 0 & 0 & 0 & 0 \\
X_{i} & 0 & 0 & 0 & 0 & -M & 0 & 0 & 0 & 0 \\
\pi_{i}^{\mathrm{T}} & 0 & 0 & 0 & 0 & 0 & J_{i} & 0 & 0 & 0 \\
B_{v i}^{\mathrm{T}} & 0 & 0 & 0 & 0 & 0 & 0 & -\beta^{2} I & 0 & 0 \\
\Omega_{i}^{\mathrm{T}} & 0 & 0 & 0 & 0 & 0 & 0 & 0 & -I & 0
\end{array}\right),
$$


where $\mathfrak{R}_{i}, \pi_{i}, J_{i}, \Omega_{i}$ and $\mu(t, i)$ are the same as the definition in Theorem 2.

On the whole, through the above certification process of two theorems and lemma, it is proved completely.

\section{Data Availability}

The datasets used and/or analyzed during the current study are available from the corresponding author on reasonable request.

\section{Conflicts of Interest}

It is declared by the authors that this article is free of conflict of interest.

\section{References}

[1] Y. Jiang and X. Li, "Broadband cancellation method in an adaptive co-site interference cancellation system," International Journal of Electronics, 2021.

[2] H. Yu, J. Tao, C. Qin et al., "A novel constrained dense convolutional autoencoder and DNN-based semi-supervised method for shield machine tunnel geological formation recognition," Mechanical Systems and Signal Processing, vol. 165, p. 108353, 2022.

[3] Y. Jin, C. Qin, J. Tao, and C. Liu, "An accurate and adaptative cutterhead torque prediction method for shield tunneling machines via adaptative residual long-short term memory network," Mechanical Systems and Signal Processing, vol. 165, p. 108312, 2022.

[4] J. Dong, Y. Cong, G. Sun, Z. Fang, and Z. Ding, "Where and how to transfer: knowledge aggregation-induced transferability perception for unsupervised domain adaptation," IEEE Transactions on Pattern Analysis and Machine Intelligence, vol. 1, 2021.

[5] Q. Li, Q. X. Zhu, S. M. Zhong, and F. L. Zhong, "Extended dissipative state estimation for uncertain discrete-time Markov jump neural networks with mixed time delays," ISA Transactions, vol. 66, pp. 200-208, 2017.

[6] Y. M. Cai, S. Y. Cai, and X. R. Mao, "Stochastic delay foraging arena predator-prey system with Markov switching," Stochastic Analysis and Applications, vol. 38, no. 2, pp. 1-22, 2019.

[7] Y. Long and G. H. Yang, "Fault detection filter design for stochastic networked control systems," International Journal of Robust and Nonlinear Control, vol. 25, no. 3, pp. 443-460, 2015.

[8] J. D. Cao, "New results concerning exponential stability and periodic solution of delayed cellular neural networks," Physics Letters A, vol. 307, no. 2-3, pp. 136-147, 2003.

[9] T. P. Chen and S. I. Amari, "Stability of asymmetric Hopfield networks," IEEE Transactions on Neural Networks, vol. 12, no. 1, pp. 159-163, 2001.

[10] J. Zhang and X. S. Jin, "Global stability analysis in delayed Hopfield neural network models," Neural Networks, vol. 13, pp. 745-753, 2000.

[11] J. Cao, "Global stability conditions for delayed CNNs," IEEE Transactions on Circuits and Systems I: Fundamental Theory and Applications, vol. 48, no. 11, pp. 1330-1333, 2001.

[12] J. Cao, "On stability of delayed cellular neural networks," Physics Letters A, vol. 261, pp. 303-308, 1999.

[13] S. H. Guo and F. L. Zhu, "Unknown input observer design for Takagi-Sugeno fuzzy stochastic system," International Journal of Control, Automation and Systems, vol. 13, no. 4, pp. $1003-$ 1009, 2015.

[14] J. Cao and J. Liang, "Boundedness and stability for CohenGrossberg neural networks with time-varying delays," Journal of Mathematical Analysis and Applications, vol. 296, pp. 665685, 2004.

[15] S. Arik, "An analysis of exponential stability of delayed neural networks with time varying delays," Neural Networks, vol. 17, pp. 1027-1031, 2004.

[16] S. Xu, J. D. Lam, and W. C. Ho, "Novel global asymptotical stability criteria for delayed cellular neural networks," IEEE Transactions on Circuits and Systems II: Express Briefs, vol. 52, pp. 349-353, 2005.

[17] H. Zhao and J. Cao, "New conditions for global exponential stability of cellular neural networks with delays," Neural Networks, vol. 18, pp. 1332-1340, 2005.

[18] Z. Wang, H. Zhang, and W. Yu, "Robust exponential stability analysis of neural networks with multiple time delays," Neurocomputing, vol. 70, pp. 2534-2543, 2007.

[19] L. Wan and J. Sun, "Mean square exponential stability of stochastic delayed Hopfield neural networks," Physics Letters A, vol. 343, pp. 306-318, 2005.

[20] Y. Sun and J. Cao, "Pth moment exponential stability of stochastic recurrent neural networks with time-varying delays," Nonlinear Analysis: Real World Applications, vol. 8, no. 4, pp. 1171-1185, 2007.

[21] Z. Wang, Y. Liu, M. Li, and X. Liu, "Stability analysis for stochastic Cohen-Grossberg neural networks with mixed time delays," IEEE Transactions on Neural Networks, vol. 17, pp. 814-820, 2006.

[22] M. Kovacic, "Timetable construction with Markovian neural network," European Journal of Operational Research, vol. 69, no. 1, pp. 92-96, 2019.

[23] P. Tino, M. Cernansky, and L. Benuskova, "Markovian architectural bias of recurrent neural networks," IEEE Transactions on Neural Networks, vol. 15, no. 1, pp. 6-15, 2004.

[24] G. Sun, Y. Cong, J. Dong, Y. Liu, Z. Ding, and H. Yu, "What and how: generalized lifelong spectral clustering via dual memory," IEEE Transactions on Pattern Analysis and Machine Intelligence, vol. 1, 2021.

[25] M. Zhang, Y. Chen, and W. Susilo, "PPO-CPQ: a privacypreserving optimization of clinical pathway query for ehealthcare systems," IEEE Internet of Things Journal, vol. 7, no. 10, pp. 10660-10672, 2020.

[26] H. Chen, Y. Miao, Y. Chen, L. Fang, L. Zeng, and J. Shi, "Intelligent model-based integrity assessment of nonstationary mechanical system," Journal of Web Engineering, vol. 20, no. 2, 2021.

[27] Z. Lv, L. Qiao, and A. K. Singh, "Fine-grained visual computing based on deep learning," ACM Transactions on Multimedia Computing Communications and Applications, vol. 17, no. 1, 2021.

[28] Z. Lv, A. K. Singh, and J. Li, "Deep learning for security problems in 5G heterogeneous networks," IEEE Network, vol. 35, no. 2, pp. 67-73, 2021.

[29] H. Tang, L. Chen, J. Lu, and C. K. Tse, “Adaptive synchronization between two complex networks with nonidentical topological structures," Physica A, vol. 387, pp. 5623-5630, 2018.

[30] S. Boyd, L. E. Ghaoui, E. Feron, and V. Balakrishnan, Linear Matrix Inequalities in System and Control Theory, SIAM, Philadelphia, PA, 1994. 